\title{
LOD 1 VS. LOD 2 - PRELIMINARY INVESTIGATIONS INTO DIFFERENCES IN MOBILE RENDERING PERFORMANCE
}

\author{
C. Ellul ${ }^{\mathrm{a}, *}, \mathrm{~J}$. Altenbuchner ${ }^{\mathrm{a}}$ \\ ${ }^{\text {a }}$ Dept. of Civil, Environmental and Geomatic Engineering, University College London, Gower Street, London, WC1E 6BT UK \\ c.ellul@ucl.ac.uk
}

Commission II WG II/2

KEY WORDS: Level of Detail, Performance, Mobile Apps

\begin{abstract}
:
The increasing availability, size and detail of 3D City Model datasets has led to a challenge when rendering such data on mobile devices. Understanding the limitations to the usability of such models on these devices is particularly important given the broadening range of applications - such as pollution or noise modelling, tourism, planning, solar potential - for which these datasets and resulting visualisations can be utilized. Much 3D City Model data is created by extrusion of 2D topographic datasets, resulting in what is known as Level of Detail (LoD) 1 buildings - with flat roofs. However, in the UK the National Mapping Agency (the Ordnance Survey, OS) is now releasing test datasets to Level of Detail (LoD) 2 - i.e. including roof structures. These datasets are designed to integrate with the LoD 1 datasets provided by the OS, and provide additional detail in particular on larger buildings and in town centres. The availability of such integrated datasets at two different Levels of Detail permits investigation into the impact of the additional roof structures (and hence the display of a more realistic 3D City Model) on rendering performance on a mobile device. This paper describes preliminary work carried out to investigate this issue, for the test area of the city of Sheffield (in the UK Midlands). The data is stored in a 3D spatial database as triangles and then extracted and served as a web-based data stream which is queried by an App developed on the mobile device (using the Android environment, Java and OpenGL for graphics). Initial tests have been carried out on two dataset sizes, for the city centre and a larger area, rendering the data onto a tablet to compare results. Results of 52 seconds for rendering LoD 1 data, and 72 seconds for LoD 1 mixed with LoD 2 data, show that the impact of LoD 2 is significant.
\end{abstract}

\section{INTRODUCTION}

The use of 3D City Models on mobile devices (such as smart phones and tablets) underpins applications including utility infrastructure validation ("call-before-you-dig"), planning (Batty et al., 2001, He et al., 2012, Coors et al., 2009), augmented reality (Chen and Chen, 2008), personalized tourist information (Schulte and Coors 2008 in (Boguslawski et al., 2011)), real estate sales and 3D navigation (Basanow et al., 2008). Importantly, $3 \mathrm{D}$ datasets are now making their way out of the research domain and into real-life usage. For example, applications such as the identification of illegal residential buildings using heat sensing devices (Watson, 2013) and the use of 3D mapping to assist town planners with noise mapping (Robertson, 2013) highlight the importance of having access to 3D data and being able to integrate the data with other information provided in $2 \mathrm{D}$, as well as display this information in a mobile context to facilitate access when visiting the sites showing illegal dwellings or noisy environments. The prevalence of these applications is growing with the increase in availability of mobile devices - indeed, there are over 1.08 billion smartphones globally (Alexander, 2012) which compares with 1.2 billion personal computers (PCs). In particular, sales of tablets are forecast to challenge those of PCs by 2017 (Arthur, 2013).

The process of extrusion 2D topographic mapping data to a given height) is an efficient method of creating the $3 \mathrm{D}$ datasets required for such applications, in particular where coverage should be city wide and high level of detail - e.g. roof structures - is not required. It results in flat-roofed buildings and also has the advantage of integrating 3D buildings with a 2D footprint (Kada, 2009). Increasingly, however, more detailed (and realistic) 3D features are becoming available, including full roof structures. Whereas these used to be limited to small blocks or individual buildings resulting from Computer Aided Design (CAD) models or terrestrial
LiDaR (Light Detection and Ranging) or other scanning, they are now being generated on a more commercial scale.

For both the flat roofs and more detailed 3D structures, the resulting 3D data is generally quite large in volume and complex in detail (Glander and Döllner, 2008) and thus potentially difficult to visualize in its entirety, in particular on a mobile device.

In order to contribute to the understanding of the performance implications of the varying levels of detail required to underpin different contexts in which 3D City Models are used, this paper compares the rendering performance obtained when visualisng a City Model using flat-roofed 3D buildings and those with more detailed roof structures. We make use of OpenGL ES (Open Graphics Library for Embedded Systems, a commonly used platform for 3D rendering in mobile devices) to develop an App for testing on a tablet Android devices.

\section{BACKGROUND}

\subsection{Generating 3D City Models}

3D City Model datasets have varying levels of detail (LoD) (Kolbe et al., 2005) - ranging from LoD 0 (a digital terrain model), through LoD 1 (a block model without any roof structures), LoD 2 (a city model having explicit roof structures and potentially associated texture) and moving up to LoD 4, which includes interior structures. Such data can be generated from digital ortho-photos, 2.5D image draping, extrusion, Computer Aided Design (CAD) models (Batty et al., 2001), LiDaR point clouds, applications such as PhotoSynth (PhotoSynth, 2012) and terrestrial Laser Scanning. Single sources of data have been used (Tse et al., 2008); however, it is more common to combine multiple sources of data (van Essen, 2008, Richmond and Romano, 2008, Wang and Sohn, 2011). 
Extrusion provides an automated method to generate a 3D model - combining 2D topographic mapping with height information derived from LiDaR data. This gives a rapid mechanism for generating an entire City Model to LoD 1.

2.1.1 Constructing Level of Detail 2 Models For more detailed modelling, it is the semi-automated methods such as photogrammetry (Ulm 2003 and Karner 2004 in (Döllner et al., 2005)) or manual modeling based on CAD or 3D modeling tools that are required (Döllner et al., 2005) and a number of authors have described approaches for LoD 2 dataset generation: Dollner and colleagues (Döllner et al., 2005) describe a process to generate a "smart building" (with continual levels of detail) starting from a block model. Sengul (Şengül, 2010) describes a process of generating 3D models using photogrammetric techniques and then converting them to CityGML. A semi-automatic approach that makes use of high resolution satellite imagery is described by (Krauß et al., 2008), although the method is limited to distinguishing between buildings with flat roofs and those with gabled roofs. The method first creates ortho-photos by combining a Digital Elevation Model with imagery, and then uses a masking approach to identify buildings by classifying them as "high, nonvegetation". Prieto and colleagues (Prieto et al., 2012) make use of point cloud data captured for individual buildings, along with post-processing using tools such as Google Sketch-up to fill in the gaps that cannot be captured by the scanning process. They also propose a manual approach to associating semantic information with the resulting 3D features. Kada (Kada, 2009) show the potential of generating a 3D model by cell decomposition, where solids are decomposed into non-intersecting, typically parameterized primitives. They then identify roof structures by first generating and then examining the normal vectors of all points inside the same cell. In all cases, these methods are semi-automated, requiring some element of manual intervention.

\section{IMPROVING PERFORMANCE - EXAMINING SYSTEM ARCHITECTURE}

Research that has been conducted into city modelling for mobile devices is perhaps limited due to the fact that devices have only recently become powerful enough to render 3D graphics. Indeed, mobile computation still faces various limitations, including limited CPU (Central Processing Unit) and memory, the absence or limited performance of graphics accelerators, the absence or limited performance of FPUs (Floating Point Units) and energy consumption issues (Nadalutti et al., 2006). When examining any issues related to computing performance, it is important to understand the hardware and software architecture of the system. This understanding leads to the identification of potential bottlenecks in the rendering process and permits optimisation where possible.

Mobile mapping is generally underpinned by a three-tier architecture (Figure 1), consisting of an end-user device or "client" (which could be a smart phone or tablet) a web server (which sends data to the client and retrieves requests from the client) and a database server, which holds the map and other data to be displayed (Mitchell, 2008). Based on this architecture, a number of considerations can be taken into account when rendering data on mobile devices: potential bottlenecks include retrieving the data from the database, transmitting the data from server to client over a mobile network (i.e. network bandwidth), and rendering the data on the device using specialist software that takes advantage of any graphics hardware. For each of these stages both hardware and software are important. In general, all through the process, it is important to minimise the amount of data in order to improve performance.

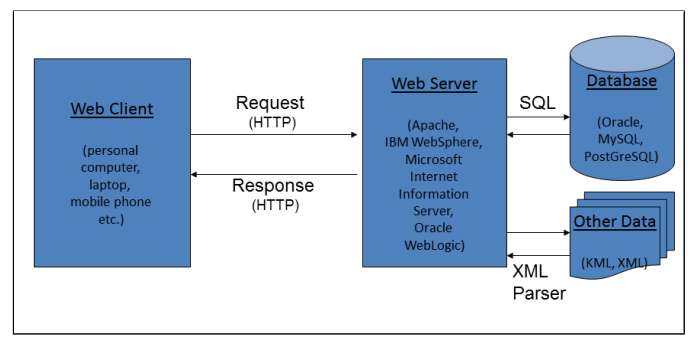

Figure 1: Mobile 3D Mapping Architecture

\subsection{Hardware Improvements}

Initial research carried out in order to overcome limitations of mobile rendering focussed on hardware (Nadalutti et al., 2006). For example (Woo et al., 2001) produced small graphic accelerator chips to allow high performance combined with low power consumption. More recently, many mobile devices have embedded graphics chips, increasingly powerful processing units (and multiple cores (NVIDIA Corporation, 2011)) and increased memory permitting improved performance and drawing them closer to the specification of gaming consoles, although battery life remains an issue to be solved (Chester, 2013).

Considering the above architecture (Figure 1), and defining rendering as the process of taking data representing the real world (i.e. the 3D City Model) and transforming it to a 2D pixel representation on screen, two main approaches to mobile mapping (including 3D mapping) can be identified - rendering data on the server side and rendering data on the client device. The latter has been made possible by the increasing specification of mobile devices.

3.1.1 Server Side Rendering For server-side rendering, data processing and rendering takes place on a powerful computer (with performance enhanced by clustering servers and using graphics accelerators) (Lamberti et al., 2003) and only the results are sent to the mobile client (Nadalutti et al., 2006, Brachtl et al., 2001), most commonly as an image or "raster". As with 2D mapping, a number of providers have generated 3D raster maps for use on mobile devices (see Figure 2 which shows 3D mapping available from Google).

3.1.2 Client Side Rendering When considering architectures where the rendering is carried out on the mobile client, the aim is to keep the graphics as simple as possible in order to achieve satisfactory performance. A number of approaches can be identified. (Döllner et al., 2005) briefly review optimization strategies such as view-frustum culling, occlusion culling, and back-face culling that operate on general graphics primitives. Additional approaches to reducing the volume of data to be rendered clientside for a 3D City Model include data compression (van Essen, 2008) and mesh simplification (Sester, 2007).

3.1.3 Combining Server Side and Client Side Approaches The results of sever side mapping are generally static (i.e a simple image, or raster). This approach has the advantage of limiting the amount of data to be downloaded, as well as allowing the images to be pre-prepared for instant rendering. However, such mapping does not provide the interactivity of a vector map, which stores the individual buildings as separate objects rather than as part of a single image. Although vector datasets can be overlaid onto 3D raster maps, they often overlap the buildings as shown in Figure 2 rather than being correctly placed. An image based approach cannot be easily navigated around or updated when underlying datasets change and does not provide click-and-identify information for the building objects. Generating a fully interactive map 
(similar to a GIS, where the user can click on a point to find out information, i.e. a vector map) (Mitchell, 2008) can be achieved by making use of the client-side rendering approaches described above, with, however, the limitation imposed by the hardware on the amount of data that can be rendered, as well as on the amount of data to be downloaded over what is frequently a low bandwidth link.

Hybrid Approaches combine server-side and client-side rendering, with the aim of limiting data to be served over the network and hence processed for rendering on the client. For example, (Prieto et al., 2012) note the importance of rendering city models on web-based platforms, and describe a process to take a detailed point cloud and transform it into a CityGML database structure. They suggest that the user can be presented with $2 \mathrm{D}$ map on which individual 3D buildings can be requested by clicking. The data is then presented as a single building in a customised JSON (JavaScript Object Notation) format (Prieto et al., 2012). Additionally, (Quillet et al., 2006) developed a system that extracts feature lines of building facades on the server side and streams data on demand to the client. The approach forces the point of view to street level. (Ellul, 2012) describes the use of topological data structures to remove shared and hidden internal walls in a model generated by extrusion, rendering the reduced dataset on the client. (Ellul and Joubran, 2012) also tested an aggregation approach, looking at the impact of generalisation on rendering performance in Google Earth.

An additional hybrid approach is proposed in this paper, where the dataset is pre-structured (triangulated) on the server in order to avoid executing a triangulation process on the client device. Common Nodes ( $x, y, z$ points) are identified server-side and reused in order to minimise the size of the dataset for transmission to the client. The proposed approach is described in further detail in Section 5. A hybrid approach requires additional processing overhead on the client side, but has the advantage of interactivity which in turn improves the usability of a 3D mobile application.

\section{TEST DATA}

The data used for testing has been provided by the Ordnance Survey (the UK National Mapping Agency) and consists of a 3D dataset for the UK city of Sheffield. The data is based on the Ordnance Survey's 2D topographic mapping dataset, Mastermap (Ordnance Survey, 2013) and covers an area of approximately $1 \mathrm{~km}$ by $1 \mathrm{~km}$ square for Sheffield. Height information is available for all buildings in the city, permitting the data to be extruded to generate LoD 1 buildings. In the center of the city, additional roof details have been added bringing the structures to LoD 2. An extract of this data (showing Mastermap topographic base mapping) is shown in Figure 3. For the area in the centre of the city shown by the darker buildings in Figure 3, LoD 2 buildings are also available. Figure 4 shows a sample of the LoD 1 and LoD 2 data.

\subsection{Summary Dataset Statistics}

For testing purposes, two datasets were extracted from the source data. Firstly a dataset covering the entire extent of the city was generated at LoD 1. A second dataset for this area was generated replacing the LoD 1 with LoD 2 data where this was available, again over the entire area. To further examine the impact of LoD 2 data on performance, a smaller subset of data was also created, covering an area of the city where LoD 2 data was almost fully available. Table 1 gives details of the resulting datasets.

Examining the data in more detail, it can be noted that the LoD 2 dataset includes some buildings not present in LoD 1 (Figure

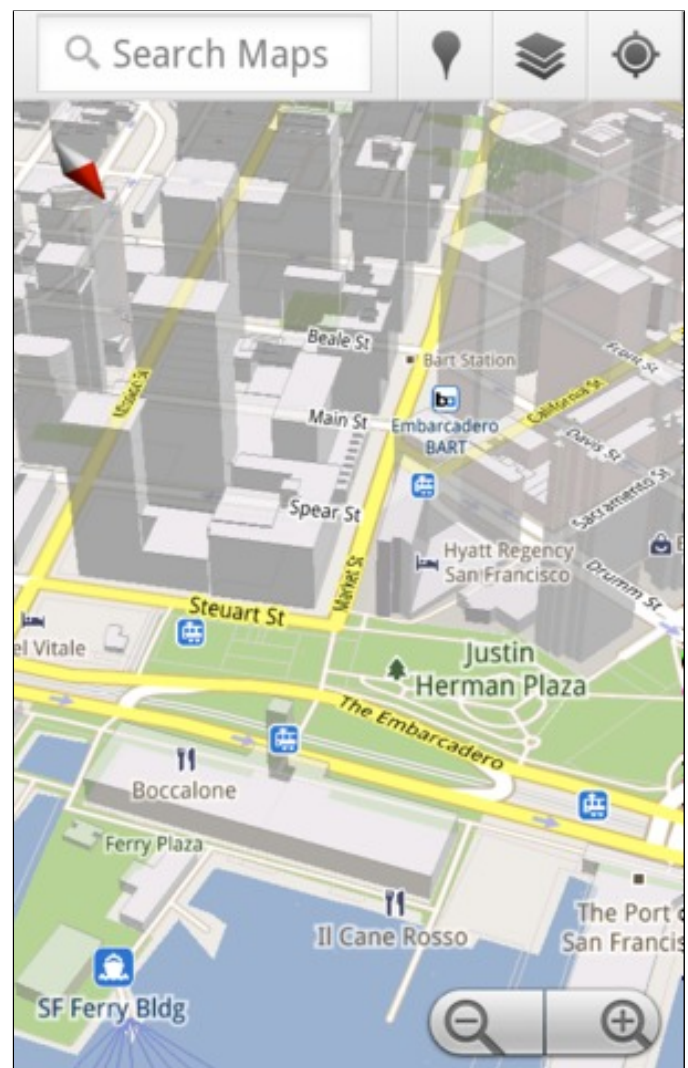

Figure 2: Google's 3D Raster Mobile Mapping source: http://www.google.co.uk/intl/en/mobile/ maps/3d/tilt.png

\begin{tabular}{|l|l|}
\hline Dataset Details & $\begin{array}{l}\text { Number of } \\
\text { Buildings }\end{array}$ \\
\hline Entire City - LoD 1 & 1560 \\
\hline Entire City - LoD 1 and 2 & 1653 \\
\hline Part City - LoD 1 & 475 \\
\hline Part City - LoD 1 and 2 & 516 \\
\hline
\end{tabular}

Table 1: Dataset Details - Sheffield

5). In the Figure, the case illustrates a greenhouse (glass house) which was included in the specification for the LoD 2 data but as it was not included in the original LoD 1 dataset as a building it was not extruded. In addition, the LoD 2 dataset has been extended slightly beyond the defined boundary to include two $3 \mathrm{D}$ buildings that serve as a test case for LoD 2 data extraction (Figure 3). To the South of the test area, the Moorfoot Building (Wikipedia, 2013) is included - this houses many services offered by Sheffield City Council and is also built with a unique step-pyramid structure. To the North West, "Park Hill" is an unusually shaped 3D structure and Europe's largest Grade II listed building (i.e. a building with national conservation importance) (The Park Hill Estate - Europes Largest Grade 2 Listed Building, 2013).

\section{IMPLEMENTING THE VISUALISATION ALGORITHM}

As briefly mentioned above, this paper proposes a hybrid approach to vector mapping of a 3D City Model. Firstly, data is prestructured into a format suitable for rendering within a database server (making use of Java algorithms to implement the required 


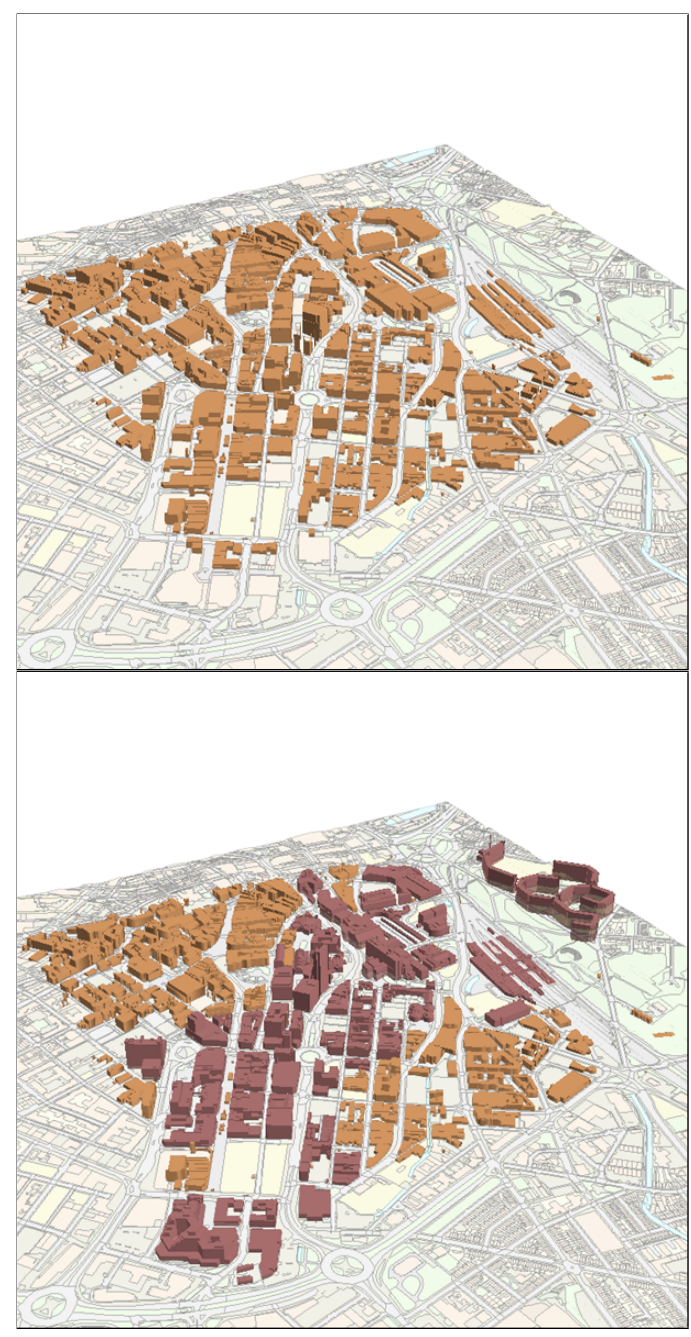

Figure 3: 3D Dataset for Sheffield, showing LoD 1 data (top) and the mix of LoD 1 and LoD 2 data (bottom)

structuring). The data can then be extracted (queried) from the spatial database using a query language such as SQL, and prepackaged for transmission as an XML dataset. The extraction and packaging operations can be implemented using a web-based programming language such as PHP. Once the data is received on the client (mobile device), the XML can then be parsed - i.e. the data elements extracted from the XML file and placed into a the required structure for rendering. As described below (Section 5.2), OpenGL ES only supports data in triangulated format. A number of data preparation stages are therefore required to convert the data into the appropriate format and structure it for rendering. These are described here, followed by a description of Open GL ES and of the implementation of the rendering software itself.

\subsection{Dataset Structuring and Transmission}

Although it is possible to triangulate the data on the fly in the App itself (Ellul and Altenbuchner, n.d.) given the quantities of data, the first step of the hybrid approach described here is to undertake the triangulation process on the server-side data.

5.1.1 Loading the Dataset into Oracle Spatial The dataset is provided in ESRI Shapefile Multi-Patch format (ESRI, 2013) and is then transformed into Oracle's Spatial Database Format (the Oracle database is used to serve the data to the test App). As part of this transformation, the dataset is fully triangulated, as required by OpenGL ES (Whitrow, 2008). Although the MultiPatch format does partially triangulate the data - specifically the

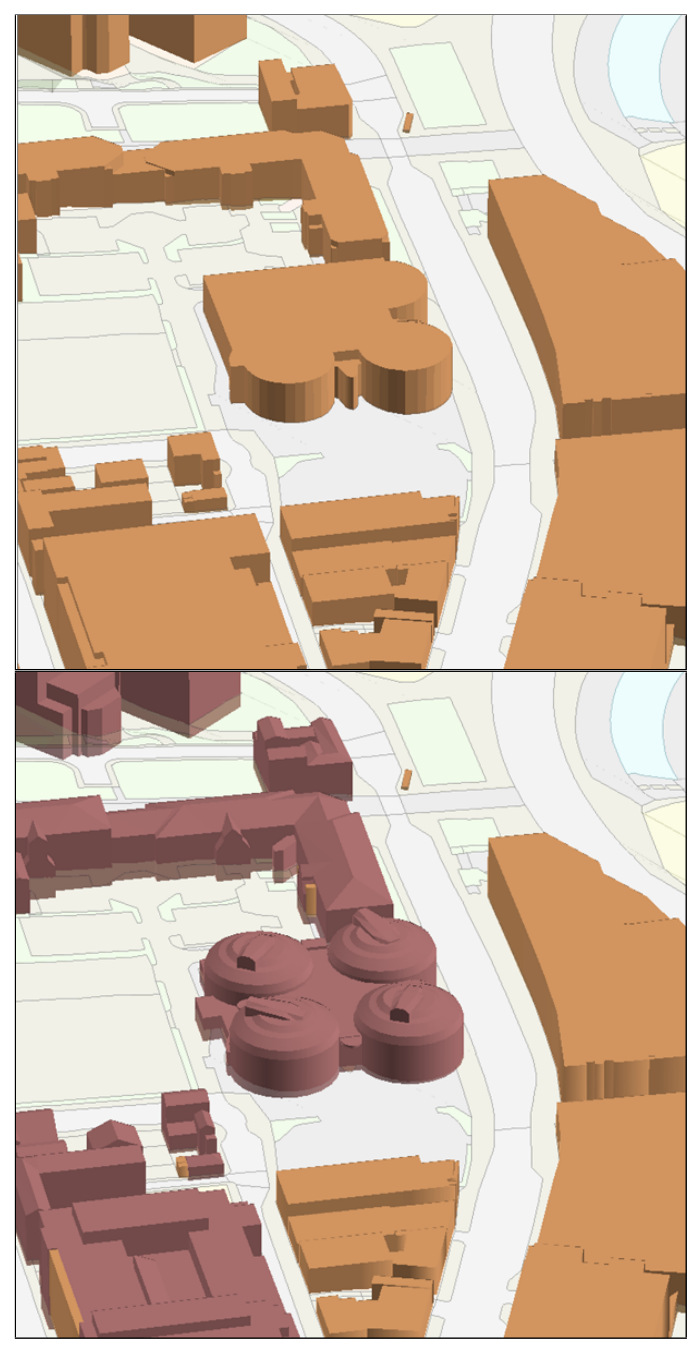

Figure 4: Comparing LoD 1 (top) and LoD 2 data (bottom) for the same buildling

walls of the building are triangulated - in the case of a standard extrusion process the roof and floor structure are stored as "geometry rings" and not suitable for rendering in OpenGL ES (ESRI, 2013). The "Feature Manipulation Engine" (FME) software from SafeSoft (SAFE Software, 2013) was used to first triangulate the data by converting it to Autodesk 3D Studio format, and then to import the resulting triangulated features into Oracle Spatial (Oracle, 2013b). Once imported into the database, the data was re-structured in preparation for visualisation.

5.1.2 Optimising the Data Structure for Rendering A number of approaches can be taken to minimise the amount of data passed through the rendering pipeline. Firstly, given that the system architecture is web based and the devices used for testing are Android Tablets and Smartphones, it is important to reduce the volume of data that is queried from the database and transferred to the device. OpenGL ES offers the option of storing all the Nodes describing the data in a single buffer. As Nodes are used by multiple triangles, this reduces the quantity of data to be transmitted. The triangles required for rendering are then constructed by making use of an indexing approach, where a list of indices point to the required Nodes. The lists of Nodes and corresponding indexes were created from the imported data by pre-processing the data in Java, extracting the triangles from the SDO_GEOMETRY objects, where a single building is made up of multiple triangles, and storing them as separate, single, triangles. The resulting data structure, which can be used directly for rendering, is as shown 
ISPRS Annals of the Photogrammetry, Remote Sensing and Spatial Information Sciences, Volume II-2/W1, ISPRS 8th 3DGeoInfo Conference \& WG II/2 Workshop, 27 - 29 November 2013, Istanbul, Turkey

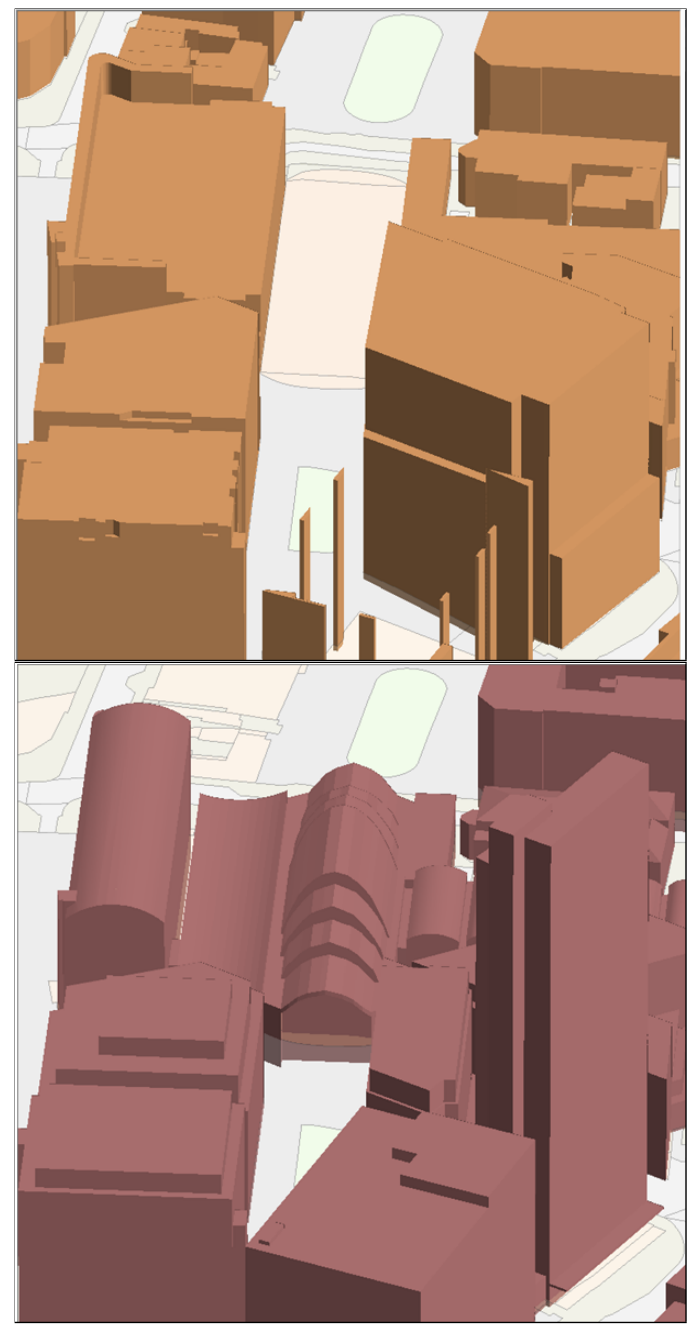

Figure 5: An example of an LoD 2 building (a glasshouse, bottom) not included in the LoD 1 dataset (top)

in the example in Table 2 and Table 3. Additional tables link the individual triangles back to the buildings.

Table 4 gives a summary of the resulting triangulated datasets, providing a first insight into the impact of LoD 2 data - the number of Nodes for the full LoD 1 and 2 mixed dataset is 1.4 times that for the LoD 1 dataset and for the part dataset, this rises to 1.6. The number of triangles for the full dataset at LoD 1 and 2 is 1.37 times that for the LoD 1 data, and for the smaller test dataset this rises to 1.56 .

\subsection{OpenGL ES Rendering}

The cross-platform and cross-language graphics Application Programmers Interface (API) OpenGL (Open Graphics Library) is a software interface to graphics hardware (McReynolds and Blythe, 2005). OpenGL has several specifications for various purposes. Of relevance here is OpenGL ES, which is commonly implemented for mobile devices . Key features were removed from

\begin{tabular}{|l|l|l|l|}
\hline Node_ID & X_Value & Y_Value $_{\text {__lValue }}$ & Z_Va $_{-}$ \\
\hline 1 & 435713.69 & 387269.31 & 55.43 \\
\hline 34 & 435731.06 & 387260 & 54.22 \\
\hline 35 & 435770.31 & 387249.75 & 52.36 \\
\hline
\end{tabular}

Table 2: Node Table Structure

\begin{tabular}{|l|l|l|}
\hline Node_ID & Triangle_ID & Node_Order \\
\hline 1 & 1 & 1 \\
\hline 34 & 1 & 2 \\
\hline 35 & 1 & 3 \\
\hline
\end{tabular}

Table 3: Index Table Structure

\begin{tabular}{|l|l|l|l|}
\hline Dataset & $\begin{array}{l}\text { Number of } \\
\text { Buildings }\end{array}$ & $\begin{array}{l}\text { Number of } \\
\text { Triangles }\end{array}$ & $\begin{array}{l}\text { Number of } \\
\text { Individual } \\
\text { Nodes }\end{array}$ \\
\hline Entire City - LoD 1 & 1560 & 81236 & 38055 \\
\hline $\begin{array}{l}\text { Entire City - LoD 1 } \\
\text { and 2 }\end{array}$ & 1653 & 115937 & 55135 \\
\hline Part City - LoD 1 & 475 & 24349 & 11421 \\
\hline $\begin{array}{l}\text { Part City - LoD 1 } \\
\text { and 2 Part }\end{array}$ & 516 & 37993 & 18202 \\
\hline
\end{tabular}

Table 4: Pre-Triangulated Data Structure, Optimised for Visualisation

OpenGL for this implementation (Anyuru, 2012)- in particular OpenGL ES only allows triangle-based surface primitives and excludes quad, quadstrip or polygon primitives. Triangles make hardware algorithms simpler and faster, as they are always convex and planar (Cozzi and Ring, 2011).

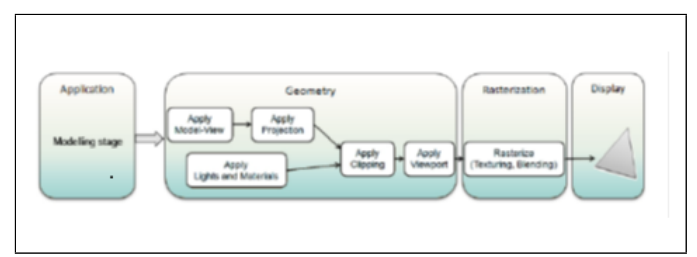

Figure 6: Rendering Pipeline after (Zechner and Green, 2011)

Figure 6 (after (Zechner and Green, 2011)) shows the path of a dataset through the rendering process in OpenGL ES. For standard rendering, the modelling software first generates a triangle mesh, which defines the shapes of the objects, and their attributes, colours, textures. Using the hybrid approach here, this is done on the server. Once transmitted, the client-side software the collates the triangles into an OpenGL readable structure (arrays containing the triangle points and index arrays describing how these are used to describe the objects to be rendered). The data structure enables OpenGL to reuse the same vertex for several triangles, reducing memory requirements on the device.

Once the data is correctly structured into arrays, a process of translation and scaling is required to ensure the 3D City Model is visible. The dataset is also scaled to ensure that the ratio between the $\mathrm{X}$ and $\mathrm{Y}$ extents matches the width:height ratio of the device (i.e. that the resulting data is not distorted). Data is then projected into a $2 \mathrm{D}$ coordinate system for display on screen, with an orthographic projection avoiding foreshortening (Smithwick and Verma, 2012) by disregarding the effect of vanishing points and applying a one to one correspondence between the real world units and the pixel positions on screen. This method is widely used for engineering applications (Whitrow, 2008).

Following projection, a "clipping" process is used to remove any objects that will not be visible to the user. For example, objects too close to the user obscure the view, and objects too far away will not be shown in enough detail to be useful. The penultimate step in rendering is transforming the resulting $2 \mathrm{D}$ data once 
again, to move from scaled real world coordinates into the screen coordinate system. The data is then ready for the final stage in the process- rasterization, which converts the data into pixels on screen (Zechner and Green, 2011).

The rendering process thus involves a number of structuring and transformation processes. It can be expected that a more complex, detailed, datasets will slow down performance.

\subsection{The Implemented Rendering Process}

The Android device selected for testing is an Acer Iconia 10-inch tablet, having a screen size of 800 by 1280 pixels, and a Dual Core, $1 \mathrm{GHz}$ processor, with $1 \mathrm{~GB}$ of RAM, running Android Version 3.2.1. As described above, for the first part of the rendering process, PHP code, combined with SQL queries, is used to generate three datasets, which are served to the Java Android App as tagged XML:

1. An ordered list of Nodes, containing the $\mathrm{X}, \mathrm{Y}$ and $\mathrm{Z}$ coordinates for each Node

2. An ordered index list, describing how the Nodes form the triangular Faces used for rendering

3. A short list detailing the geometrical extents of the dataset i.e. the minimum bounding volume. This is used to ensure that the resulting data is centred on the device's screen when rendered.

Traditionally, the XML may be generated by iterating through each row of the dataset, generating the required tagged data using string concatenation commands. However, Oracle offers both "XMLAGG" - which aggregates all rows of a query into one document - and the "XMLEMENT" - which wraps individual data items in XML tags - query statements to automatically generate a full XML document from a dataset without the need for iteration (Oracle, 2013a).

Once the XML data is parsed on the client device, it then passes through the OpenGL rendering pipeline described above. To transform the real-world coordinates (which are in British National Grid) into values appropriate for display, the initial screen coordinate system was set up with its origin $(0,0,0)$ in the centre of the viewport. The real world coordinate values of the imported buildings are then transformed into screen coordinates by finding the centre point of each axis for the dataset (e.g. $(x \max +x \operatorname{xin}) / 2)$ and then translating the dataset by the respective distance along the negative $\mathrm{x}$ and $\mathrm{y}$. The $\mathrm{z}$-axis is shifted upwards. For the second and third steps of the rendering process, OpenGL ES uses the bounding coordinates of the dataset to project the 3D triangles into 2D space and to set clipping boundaries, which in the case of this project are set to the extents of the dataset. The near and far clipping planes were set to 1000 and -1000 in order to avoid clipping when the user pans the objects in the z-direction. Projection is set to be orthographic.

Time measurements are taken at various stages through the process and written to the device as a CSV file in order to permit performance comparisons.

\section{RESULTS}

In total ten rendering time measurements were carried out for each of the four test datasets. The results were then averaged for each dataset. Figure 7 shows the resulting rendering times, with

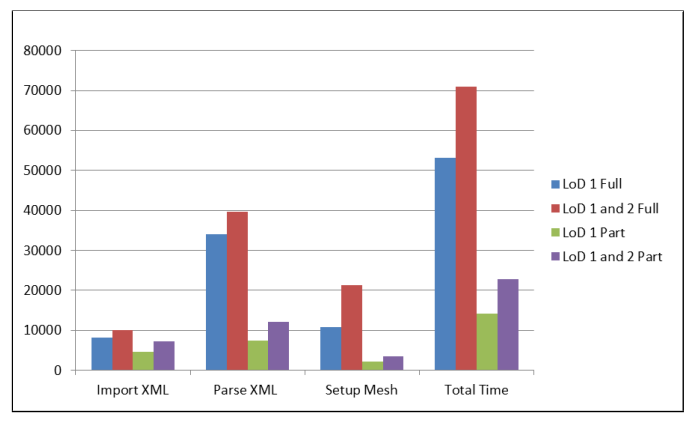

Figure 7: Rendering Results for All Datasets

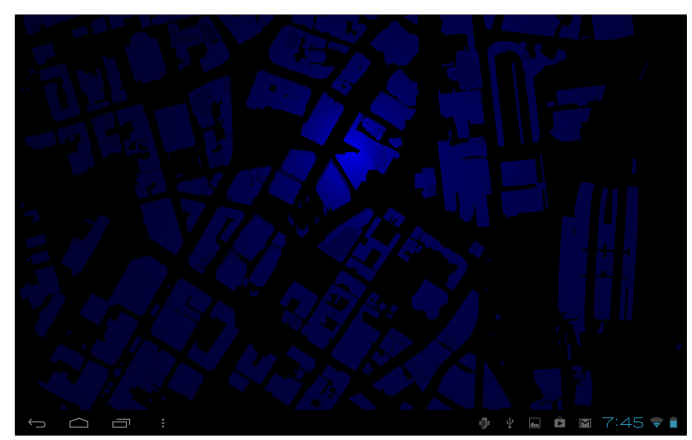

Figure 8: Sheffield - LoD 1 Dataset - Aerial View

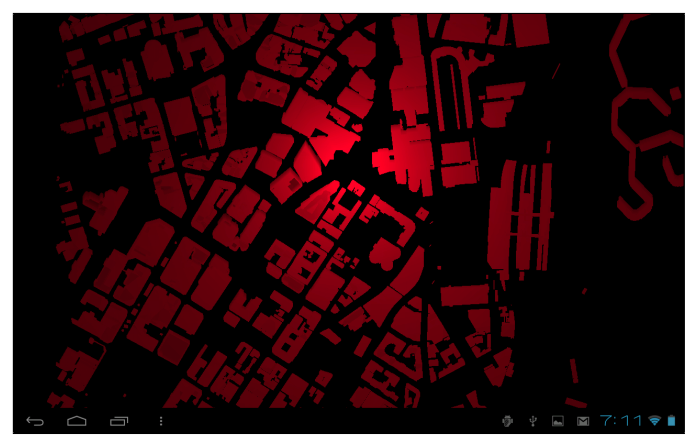

Figure 9: Sheffield - LoD 1/2 Dataset - Aerial View

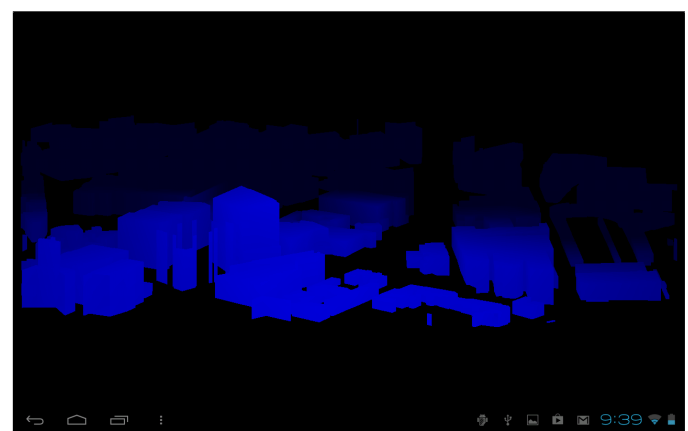

Figure 10: Sheffield - LoD 1 Dataset - City View

time (in milli-seconds) given in Table 5. Figures 8, 9, 10 and 11 show the resulting rendered datasets.

In Table 5, the "Import XML" time refers to the time to convert the dataset into XML from the database using PHP and download the dataset to the mobile device. The "Parse XML" time refers to the time required to extract the data values from the XML, and the "Setup Mesh" time refers to the time required to convert the extracted data into the required format for OpenGL rendering. "Total Time" includes all these factors and other elements of tim- 


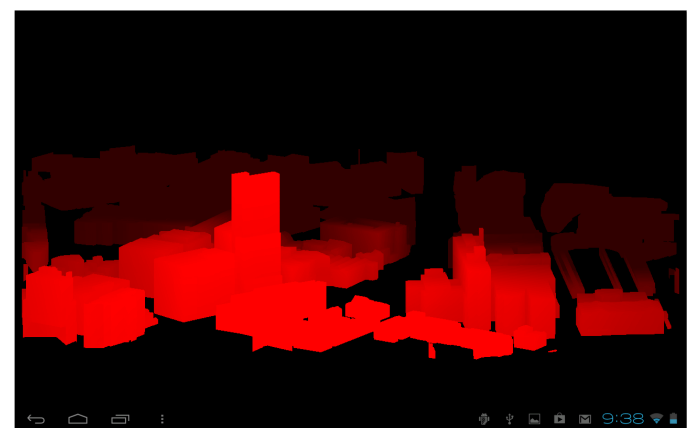

Figure 11: Sheffield - LoD 1/2 Dataset - City View

\begin{tabular}{|l|l|l|l|l|}
\hline Dataset & $\begin{array}{l}\text { Import } \\
\text { XML }\end{array}$ & $\begin{array}{l}\text { Parse } \\
\text { XML }\end{array}$ & $\begin{array}{l}\text { Setup } \\
\text { Mesh }\end{array}$ & $\begin{array}{l}\text { Total } \\
\text { Time }\end{array}$ \\
\hline LoD 1 All & 8209 & 34127.2 & 10807.1 & 53213.3 \\
\hline $\begin{array}{l}\text { LoD 1 and } \\
\text { LoD 2 All }\end{array}$ & 9981.8 & 39714.8 & 21221 & 70967.4 \\
\hline LoD 1 Part & 4674.9 & 7340.9 & 2195.4 & 14237.3 \\
\hline $\begin{array}{l}\text { LoD 1 and } \\
\text { LoD 2 Part }\end{array}$ & 7213.6 & 12156.8 & 3492.1 & 22888.2 \\
\hline
\end{tabular}

Table 5: Results - All Times in milliseconds

ing (e.g. to change activity from download to rendering), and all times are measured in milli-seconds.

\section{DISCUSSION AND FURTHER WORK}

The results obtained, and in particular the overall rendering time of 52 seconds for the LoD 1 data and 72 seconds for the mixed LoD 1 and 2 data, highlight the potential of the hybrid approach for 3D City Model rendering, where part of the dataset restructuring is carried out as a pre-prepared dataset on the server, reducing the computational effort (and specifically the triangulation process) required on the mobile device. In addition, the results bring to the fore the importance of selecting an appropriate dataset for rendering and visualisation on mobile devices, with the mixed LoD 1/2 dataset covering the entire Sheffield area taking 1.3 times longer to render than the LoD 1 dataset. This increases to 1.6 times when the smaller, mostly LoD 2, dataset is considered.

For all the four datasets, the parsing of the XML constitutes a significant proportion of the overall rendering time (between 50 and $64 \%$ ). However, the significance of the dataset import time (i.e. the time to download the dataset from the server) varies for the larger dataset, it is approximately $15 \%$ of the overall time, rising to $31 \%$ for the smaller datasets. Some of this variation may be due to varying network bandwidth, which was not measured separately and may also be a bottleneck for performance. Conversley, mesh set-up time (i.e. the time to convert the downloaded data into the required OpenGL arrays - amounts to approximately $25 \%$ for the larger dataset, but only $15 \%$ for the smaller datasets. Considering the number of triangles, for the mixed datasets (LoD 1 and LoD 2, both full and partial) the data is represented by around 70 triangles per building. This is reduced to around 51 for the LoD 1 dataset. Figure 12 shows these ratios.

Some considerations should be made when reviewing these results. As noted above, there were a number of additional buildings included in the LoD 2 datasets that did not form part of the LoD 1 data - this will have resulted in a slightly increased overall

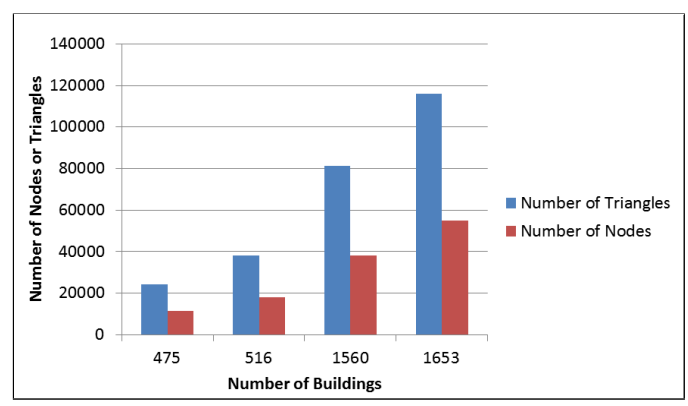

Figure 12: Node and Triangle to Building Count Ratios

rendering time for the LoD 1/2 tests, as well as increased numbers of nodes and triangles. The triangulation algorithm used to prepare the datasets was that provided by Safesoft's FME product (SAFE Software, 2013) and may have generated a larger number of triangles than would be produced using other methods.

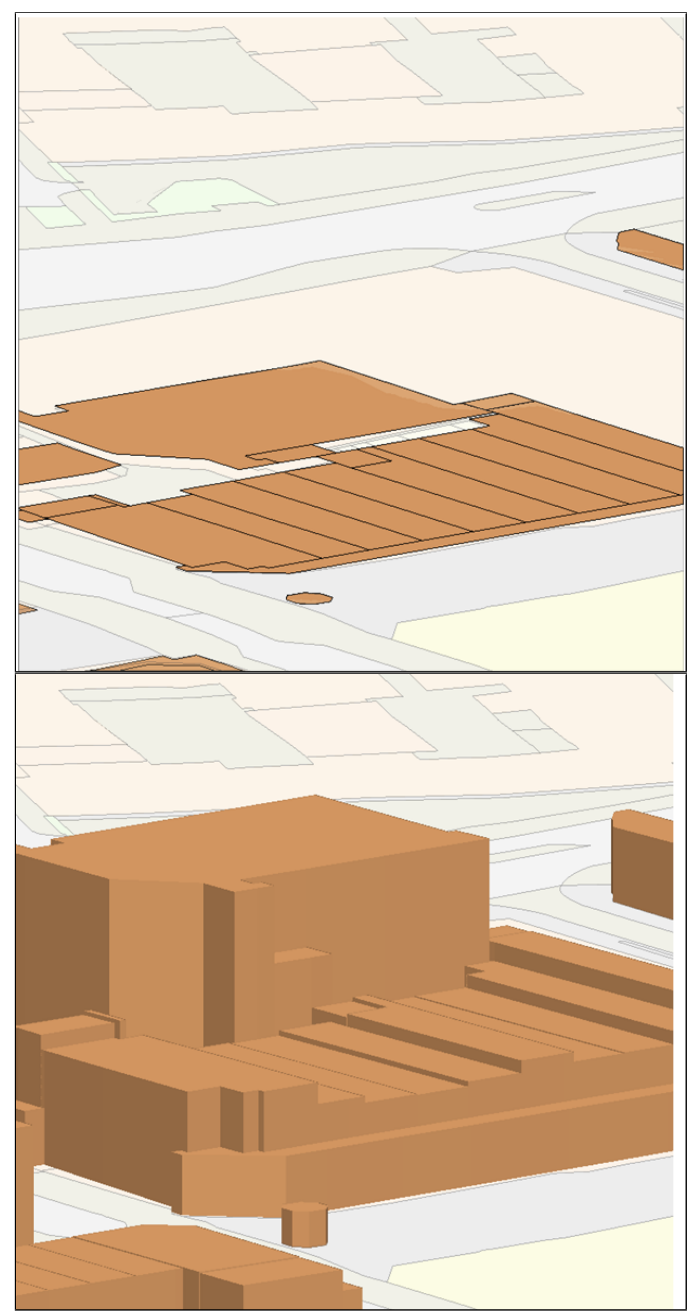

Figure 13: An extract of the Sheffield Dataset showing that Internal Walls are also Rendered

A number of areas for further development and testing can also be suggested. Firstly, as shown in Figure 13, the dataset used for these tests also includes internal walls, which are not visible to the end user when viewing the data at city scale. These could be removed (via a process of aggregation or generalisation) to reduce the number of triangles to be rendered. Secondly, the precreation of the triangles for rendering potentially resulted in overall performance improvements. However, this currently means 
that the triangles are de-coupled from the original data, meaning that changes in the latter will not be reflected. The "Ear Clipping" triangulation algorithm is a simple and robust algorithm which works well for geospatial polygons (Cozzi and Ring, 2011). It is underpinned by an assumption that the polygons which represent the building roofs in spatial datasets are "simple polygons" (i.e. do not have internal holes or self-intersections), iterating around the Nodes making up the polygon removing each triangular "ear" as it goes. Triggers could be developed inside the Oracle database to automatically re-triangulate the data when the underlying buildings change.

An additional dataset for the UK city of Newcastle is available and should be used to validate the results obtained for these preliminary tests. In addition, having access to additional data will permit further investigation into the ratios of Nodes and Triangles present in LoD 1 and LoD 2 buildings. As can be seen in Figure 3 the data is currently very much intermingled, which makes separating out the different LoD buildings difficult in a 3D context as the required spatial queries are not available due to the lack of a Spatial Reference ID for 3D British National Grid data in Oracle. Spatial queries in 2D could be used to overcome this issue.

The results do show a significant difference in rendering time with increasing level of detail - but they also highlight that rendering a city-wide dataset at LoD 1 can be slow - 53 seconds not being considered acceptable response time in a web-based situation (Marsh and Haklay, 2010). Further work is thus required on both server-side and client-side aspects of rendering, perhaps combining the work described here with additional prepreparation of datasets and implementation of the techniques described in Section 3 such as data compression (van Essen, 2008) and mesh simplification (Sester, 2007) over a block of buildings to test for optimal performance. Techniques to reduce the volume of transmitted data and the time required to parse this data could also be considered - for example, (Crockford, 2006) proposes Javascript Object Notation (JSON) as a smaller footprint data transmission mechanism.

The adequacy of 3D detail, the visual impact of the resulting 3D dataset, the suitability of the response times and the overall usability of the 3D model is, perhaps, more subjective and will depend on the specific application for which the 3D City Model is to be used i.e. the context of use (Kjeldskov and Graham, n.d.). For example, definitions such as "an application should respond within 2 seconds to provide users with a feeling of interactivity" cannot be applied universally and in mobile applications, 2 seconds is too long for an application that communicates with a driver (Marsh and Haklay, 2010). The scale at which the data is visualised is a second factor to consider. The source dataset contains a very high level of detail with buildings subdivided into smaller elements where roof height varies. This detail may be useful, for example, in a planning context, for large scale mapping. However, when a larger extent of the dataset is viewed (as in Figure 8 and Figure 9 above) the detail perhaps appears to "clutter" the map and the more simple lines of an aggregated and simplified dataset could be said to be more visually appealing, without significant loss of detail or general shape of the overall city. An aggregated dataset may, therefore, be suitable for visualising data at a smaller scale for example, to visualise 3D air quality distribution across a city. Therefore the methods developed should be extended to make better use of the web-based approach to querying and downloading data, dynamically tracking the user's interaction with the data and retrieving detailed (LoD 2) data only when the user is zoomed in.

More generally, the datasets used for this test, the multiple methods used to generate 3D data, the increasing availability of Build- ing Information Models (BIM, which go beyond LoD 4 to model a digital representation of physical and functional characteristics as a shared resource to be used throughout a building's life-cycle (Smith, 2011), and are now becoming mandatory in large government construction in the UK (Ngo, 2012)) and the wide range of potential applications that make use of 3D mobile mapping and City Models all highlight the fact that it is unlikely that users will be working with or require a dataset composed of a single level of detail - indeed, as discussed above the detail should be adapted to the specific application and context of use. This in turn highlights the requirement for further investigation into the specific detail and model sizes (number of Triangles and Nodes) generated when using the each of the methods described in Section 2.1.1 to generate 3D data. The potential of 3D generalisation to provide more context (application) specific data and to address performance issues on mobile devices should also be investigated, to ensure that the data provided is contextually appropriate and presented at a scale that is relevant while meeting expected performance.

\section{ACKNOWLEDGELMETNS}

The authors would like to thank the Ordnance Survey for the use of both the LoD 1 dataset (Mastermap Topographic Mapping) and the LoD 2 dataset for the Sheffield and area of the UK. Additional thanks go to Safe Software for the use of FME (SAFE Software, 2013).

\section{REFERENCES}

Alexander, A., 2012. Smartphone usage statistics 2012.

Anyuru, A., 2012. Professional WebGL Programming: Developing 3D Graphics for the Web. Wrox.

Arthur, C., 2013. Tablets will challenge pc sales by 2017 as android passes ipad, says idc.

Basanow, J., Neis, P., Neubauer, S., Schilling, A. and Zipf, A., 2008. Towards $3 \mathrm{~d}$ spatial data infrastructures (3d-sdi) based on open standardsexperiences, results and future issues. In: Advances in 3D Geoinformation Systems, Springer, pp. 65-86.

Batty, M., Chapman, D., Evans, S., Haklay, M., Keupers, S., Shiode, N., Hudson Smith, A. and P, T., 2001. Visualising the city: Communicating urban design to planners and decisionmakers. Planning Support Systems, Models and Visualisation Tools pp. $405-443$.

Boguslawski, P., Gold, C. and Ledoux, H., 2011. Modelling and Analysing 3D Buildings with a Primal/Dual data structure. ISPRS Journal of Photogrammetry and Remote Sensing 66, pp. 188-197.

Brachtl, M., Šlajs, J. and Slavık, P., 2001. Pda based navigation system for a 3d environment. Computers \& Graphics 25(4), pp. 627-634.

Chen, J. and Chen, C., 2008. Foundations of 3D Graphics Programming: Using JOGL and Java3D, 2nd Edition.

Chester, E., 2013. Next generation mobile processors will outperform PS3 and Xbox 360.

Coors, V., Hünlich, K. and On, G., 2009. Constraint-based generation and visualization of 3D city models. In: 3D GeoInformation Sciences, Springer, pp. 365-378.

Cozzi, P. and Ring, K., 2011. 3D engine design for virtual globes. AK Peters Limited. 


\section{Crockford, D., 2006. JSON: The fat-free alternative to XML.}

Döllner, J., Buchholz, H., Brodersen, F., Glander, T., Jütterschenke, S. and Klimetschek, A., 2005. Smart Buildings-A concept for ad-hoc creation and refinement of 3D building models. In: Proceedings of the 1st International Workshop of 3D City Models, Bonn, Germany.

Ellul, C., 2012. Can Topological Pre-Culling of Faces Improve Rendering Performance of City Models in Google Earth? Progress and Trends in 3D GeoInformation Sciences -Lecture Notes in Geoinformation and Cartography pp. 133-154.

Ellul, C. and Altenbuchner, J., n.d. Investigating Approaches to Improving Rendering Performance of 3D City Models on Mobile Devices, Manuscript Under Review. GeoSpatial Information Science.

Ellul, C. and Joubran, J., 2012. Preliminary investigations into the potential of improving rendering performance of 3D datasets using 2D generalisation. In: Usage, Usability, and Utility of 3D City Models-European COST Action TU0801, Proceedings of the conference held 29-31 October, 2012 in Nantes, France. Edited by Th. Leduc, G. Moreau, and R. Billens, id. 03005, 7 pp., Vol. 1, p. 03005.

ESRI, 2013. The multipatch geometry type. http://www . esri.com/library/whitepapers/pdfs/multipatchgeometry-type.pdf.

Glander, T. and Döllner, J., 2008. Techniques for generalizing building geometry of complex virtual $3 \mathrm{~d}$ city models. In: Advances in 3D Geoinformation Systems, Springer, pp. 381-400.

He, S., Moreau, G. and Martin, J.-Y., 2012. Footprint-based 3d generalization of building groups for virtual city visualization. In: GEOProcessing 2012, The Fourth International Conference on Advanced Geographic Information Systems, Applications, and Services, pp. 177-182.

Kada, M., 2009. The $3 \mathrm{~d}$ berlin project. In: Photogrammetric week, pp. 331-340.

Kjeldskov, J. and Graham, C., n.d. A review of mobile HCI research methods.

Kolbe, T. H., Gröger, G. and Plümer, L., 2005. CityGML: Interoperable access to 3D city models. In: Geo-information for disaster management, Springer, pp. 883-899.

Krauß, T., Lehner, M. and Reinartz, P., 2008. Generation of coarse 3D models of urban areas from high resolution stereo satellite images. The International Archives of Photogrammetry, Remote Sensing and Spatial Information Sciences 37, pp. 10911098 .

Lamberti, F., Zunino, C., Sanna, A., Fiume, A. and Maniezzo, M., 2003. An accelerated remote graphics architecture for pdas. In: Proceedings of the eighth international conference on 3D Web technology, ACM, pp. 55-ff.

Marsh, S. and Haklay, M., 2010. Evaluation and deployment.

McReynolds, T. and Blythe, D., 2005. Advanced graphics programming using OpenGL. Morgan Kaufmann.

Mitchell, T., 2008. Web mapping illustrated: Using Open Source GIS toolkits. O'Reilly Media.

Nadalutti, D., Chittaro, L. and Buttussi, F., 2006. Rendering of $\mathrm{x} 3 \mathrm{~d}$ content on mobile devices with opengl es. In: Proceedings of the eleventh international conference on $3 \mathrm{D}$ web technology, ACM, pp. 19-26.
Ngo, M. H., 2012. UK Construction Industrys responses to Government Construction Strategy BIM deadline and applications to civil engineering education. https:// workspace.imperial.ac.uk/civilengineering/Public/ technicalpapergroupA/18A-Ngo-UKConstructionInd. ResponsestoGovernmentConstructionStrategy2.pdf.

NVIDIA Corporation, 2011. The Benefits of Quad Core CPUs in Mobile Devices. http://www.nvidia.co.uk/content/PDF/ tegra_white_papers/tegra-whitepaper-0911a.pdf.

Oracle, 2013a. Generating XML Data from the Database. http://docs.oracle.com/cd/B28359_01/appdev.111/ b28369/xdb13gen.htm.

Oracle, 2013b. Oracle Spatial and Oracle Locator. http://www.oracle.com/uk/products/database/ options/spatial/index.html.

Ordnance Survey, 2013. Ordnance Survey Mastermap. http: //www.ordnancesurvey.co.uk/oswebsite/products/osmastermap/index.html.

PhotoSynth, 2012. About photosynth. http://photosynth. net/about.aspx.

Prieto, I., Izkara, J. and Delgado, F., 2012. From point cloud to web 3D through CityGML. In: Virtual Systems and Multimedia (VSMM), 2012 18th International Conference on, IEEE, pp. 405412.

Quillet, J.-C., Thomas, G., Granier, X., Guitton, P. and Marvie, J.-E., 2006. Using expressive rendering for remote visualization of large city models. In: Proceedings of the eleventh international conference on 3D web technology, ACM, pp. 27-35.

Richmond, P. and Romano, D., 2008. Automatic generation of residential areas using geo-demographics. In: Advances in 3D Geoinformation Systems, Springer, pp. 401-416.

Robertson, A., 2013. 3D city planning tool maps noise pollution as visible data. http://www.theverge.com/2012/4/2/ 2920286/3d-city-planning-tool-noise-pollutionfraunhofer-institute.

SAFE Software, 2013. FME - Break Down the Barriers to Using and Sharing Data. http://www.safe.com/fme/fmetechnology/.

Şengül, A., 2010. Extracting semantic building models from aerial stereo images and conversion to citygml.

Sester, M., 2007. 3d visualization and generalization. In: Photogrammetric Week, Vol. 7, pp. 03-09.

Smith, M., 2011. BIM in construction. National Building Specification (NBS). http://www.thenbs.com/topics/bim/ articles/bimInConstruction. asp.

Smithwick, R. and Verma, M., 2012. Pro OpenGL ES for Android. Apress, New York,

The Park Hill Estate - Europes Largest Grade 2 Listed Building, 2013. http://www.bbc.co.uk/learningzone/clips/thepark-hill-estate-sheffield-europes-largestgrade-2-listed-building/6427.html.

Tse, R., Gold, C. and Kidner, D., 2008. 3D City Modelling from LiDaR Data. In: Advances in 3D Geoinformation Systems, Springer, pp. 161-175.

van Essen, R., 2008. Maps get real: Digital maps evolving from mathematical line graphs to virtual reality models. In: Advances in 3D Geoinformation Systems, Springer, pp. 3-18. 
ISPRS Annals of the Photogrammetry, Remote Sensing and Spatial Information Sciences, Volume II-2/W1, ISPRS 8th 3DGeolnfo Conference \& WG II/2 Workshop, 27 - 29 November 2013, Istanbul, Turkey

Wang, L. and Sohn, G., 2011. An integrated framework for reconstructing full $3 \mathrm{~d}$ building models. In: Advances in 3D GeoInformation Sciences, Springer, pp. 261-274.

Watson, L., 2013. Council spends 24,000 on spy plane with thermal imaging cameras to catch immigrants living illegally in 'sheds with beds'. http://www.dailymail.co. uk/news/article-2346759/Council-uses-spy-planethermal-imaging-cameras-catch-immigrants-livingillegally-garden-sheds.html.

Whitrow, R., 2008. OpenGL graphics through applications. Springer, New York,

Wikipedia, 2013. http://en.wikipedia.org/wiki/ Moorfoot_Building.

Woo, R., Yoon, C.-W., Kook, J., Lee, S.-J., Lee, K., Park, Y.H. and Yoo, H.-J., 2001. A $120 \mathrm{~mW}$ embedded 3D graphics rendering engine with $6 \mathrm{Mb}$ logically local frame-buffer and 3.2 GByte/s run-time reconfigurable bus for PDA-chip. In: VLSI Circuits, 2001. Digest of Technical Papers. 2001 Symposium on, IEEE, pp. 95-98.

Zechner, M. and Green, R., 2011. Beginning Android 4 Games Development. Apress. 